\title{
Mechanism of Superhyperfine Structure in $\mathrm{SnO}_{2}: \mathrm{V}^{4+} \dagger$
}

\author{
I. Chen, C. Kikuchi, and H. Watanabe* \\ Department of Nuclear Engineering, University of Michigan, Ann Arbor, Michigan
}

(Received 31 July 1964)

\begin{abstract}
Molecular orbital theory has been applied to $\mathrm{SnO}_{2}: \mathrm{V}^{4+}$ to account for the large and small superhyperfine structure observed by From $e t$ al. The unpaired spin density at the next-nearest ligand tin nucleus is formulated. The result can be intepreted as due to two electron-transfer processes. The first comes from the formation of antibonding molecular orbital. The second arises from the configuration mixing. The first process is found to be dominant and is proportional to the square of overlap integral between vanadium $3 d$ and tin $5 s$ orbitals. Calculated ratio of the large and small superhyperfine structure is in good agreement with experiment.
\end{abstract}

\section{INTRODUCTION}

$\mathrm{Qu}^{\mathrm{n}}$ UPERHYPERFINE structure (shfs) or transferred hyperfine structure is an anomalous hyperfine structure observed in electron spin resonance (ESR) spectra of many transition-metal-ion complexes ${ }^{1}$ and is interpreted as resulting from the interaction of unpaired electrons with ligand nuclear spins. It constitutes one of the most important evidences that the unpaired electrons in complexes are delocalized and, hence, provides information about an effect that is very difficult to obtain from first principle calculation. In complexes with large covalent character, shfs due to next-nearest ligands have been observed, such as in AIIBvI compounds containing $\mathrm{Mn}^{2+}$ ions'; rutile containing $\mathrm{Mo}^{5+}$ ions ${ }^{3}$; and tin oxide containing $\mathrm{V}^{\mathrm{q}+}$ ions. ${ }^{4,5}$

The problem we wish to discuss in this paper is the mechanism of shfs as inferred from the large and small shfs observed in the ESR spectrum of $\mathrm{SnO}_{2}: \mathrm{V}^{4+}$ mentioned in the companion paper.

\section{MOLECULAR ORBITAL TREATMENT}

The theory of shfs for the nearest-neighbor ligands for $\mathrm{Mn}^{2+}$ ions in the rutile structure $\mathrm{ZnF}_{2}$ has been considered by Keffer et al., ${ }^{6}$ and by Clogston and his co-workers. ${ }^{7}$ The purpose of this paper is to extend the theory to the second-nearest ligands. We are concerned with the isotropic shfs, which is proportional to the unpaired electron density at the ligand nuclei.

\footnotetext{
+ Supported in part by National Aeronautics and Space Administration Grant.

* Permanent address: Department of Physics, Hokkaido University, Sapporo, Hokkaido, Japan.

1 J. H. E. Griffiths, J. Owen, and I. M. Ward, Proc. Roy. Soc. (London) A219, 526 (1953); M. Tinkham; ibid. A236, 535, 549 (1956).

${ }^{2} \mathrm{~J}$. Lambe and C. Kikuchi, Phys. Rev. 119, 1256 (1960);

T. P. P. Hall, W. Hayes, and F. I. B. Williams, Proc. Phys. Soc. (London) A78, 883 (1961) ; P. B. Dorain, Phys. Rev. 112, 1058 (1958); J. Schneider, S. R. Sircar, and A. Rauber, Z. Naturforsh. $18 \mathrm{a}, 980$ (1963).

3 T. Chang, Bull. Am. Phys. Soc. 8, 464 (1963)

4. H. Kasai, Phys. Letters 7, 5 (1963).

5 W. H. From, C. Kikuchi, and P. Dorain, Bull. Am. Phys. Soc. 9, 37 (1964).

${ }^{6}$ F. Keffer, T. Oguchi, W. O'Sullivan, and J. Yamashita, Phys. Rev. 115, 1553 (1959).

${ }^{7}$ A. M. Clogston, J. P. Gordon, V. Jaccarino, M. Peter, and

L. R. Walker, Phys. Rev, 117, 1222 (1960).
}

Molecular orbitals (MO) are constructed from the linear combinations of (i) vanadium $3 d$ orbital $U_{v}$, (ii) nearest ligand oxygen orbital $U_{0}$, and (iii) nextnearest ligand tin orbital $U_{s}$. The three orthonormal MO's are of the form

$$
\begin{aligned}
& \psi_{a}=U_{v}+\beta_{a} U_{0}+\gamma_{a} U_{s}, \\
& \psi_{b}=\alpha_{b} U_{v}+U_{0}+\gamma_{b} U_{s}, \\
& \psi_{c}=\alpha_{c} U_{v}+\beta_{c} U_{0}+U_{s},
\end{aligned}
$$

where the coefficients $\alpha, \beta$, and $\gamma$ are assumed to be real and are small comparable in magnitude to the overlap integrals $S_{v 0} \equiv\left(U_{v} \mid U_{0}\right)$. A schematic diagram of energy levels is given in Fig. 1.

From the orthogonality of the MO's, we have

$$
\begin{aligned}
& \beta_{a}=-\left(S_{v 0}+\alpha_{b}\right), \\
& \gamma_{a}=-\left(S_{v 8}+\alpha_{c}\right), \\
& \gamma_{b}=-\left(S_{0 s}+\beta_{c}\right) .
\end{aligned}
$$

The coefficients $\alpha_{b}, \alpha_{c}$, and $\beta_{c}$ can be obtained from the secular equation

$$
\sum_{\nu}\left(H_{\mu \nu}-S_{\mu \nu} E_{i}\right) C_{i \nu}=0 \quad(\mu, \nu=v, 0, s ; i=a, b, c),
$$

where $H_{\mu \nu}$ is the matrix element of the effective oneelectron Hamiltonian between the two AO's, $U_{\mu}$ and $U_{\nu} ; C_{i}$ is the coefficient of AO $U_{\nu}$ in the MO $\psi_{i}$.

Denoting the energies of MO's $\psi_{a}, \psi_{b}$, and $\psi_{c}$ by $E_{a}$, $E_{b}$, and $E_{c}$, respectively, we have from Eq. (7),

$$
\begin{aligned}
\alpha_{b}=\left(S_{v 0} E_{b}-H_{v 0}\right) / & \left(H_{v v}-E_{b}\right) \\
& =S_{v 0}\left[\left(E_{b}-K_{v 0}\right) /\left(H_{v v}-E_{b}\right)\right], \\
\alpha_{c}=\left(S_{v s} E_{c}-H_{v s}\right) /( & \left.H_{v v}-E_{c}\right) \\
= & S_{v s}\left[\left(E_{c}-K_{v s}\right) /\left(H_{v v}-E_{c}\right)\right], \\
\beta_{c}=\left(S_{0 s} E_{c}-H_{0 s}\right) /( & \left.H_{00}-E_{c}\right) \\
= & S_{0 s}\left[\left(E_{c}-K_{0 s}\right) /\left(H_{00}-E_{c}\right)\right],
\end{aligned}
$$

where $H_{\mu \nu}\left(\mu \neq \nu_{\nu}\right)$ is assumed proportional to $S_{\mu \nu}$, with $K_{\mu \nu}$ as the proportionality factor. 
The lowest and the next-lowest energy configurations of this three-electron system are given by the Slater determinants

and

$$
\Psi_{1}=\left\{\psi_{a^{+}}, \psi_{b}{ }^{+}, \psi_{b}^{-}\right\}
$$

$$
\Psi_{2}=\left\{\psi_{a}^{+}, \psi_{b}+, \psi_{a}^{-}\right\},
$$

where the superscripts + and - indicate the spin functions. In the second configuration an electron is transferred from the filled MO $\psi_{b}$ to the unpaired MO $\psi_{a}$. The ground-state wavefunction of this system can be written as the linear combination of the two configurations:

$$
\begin{aligned}
\Psi & =\Psi_{1}+\lambda \Psi_{2} \\
& =\left[\psi_{a}^{+}, \psi_{b}{ }^{+},\left(\psi_{b}+\lambda \psi_{a}\right)^{-}\right] .
\end{aligned}
$$

The unpaired spin density at $\mathbf{r}, \rho_{s}(\mathbf{r})$, for this state can be written as

$$
\rho_{s}(\mathbf{r})=\left|\psi_{a}(\mathbf{r})\right|^{2}+\left|\psi_{b}(\mathbf{r})\right|^{2}-\left|\psi_{a}(\mathbf{r})+\lambda \psi_{b}(\mathbf{r})\right|^{2}
$$

At the nucleus of the next-nearest ligand $\mathrm{Sn}, \mathbf{r}=\mathbf{r}_{\boldsymbol{s}}$,

hence,

$$
\psi_{a}\left(\mathbf{r}_{s}\right) \approx \gamma_{a} U_{s}(0), \quad \psi_{b}\left(\mathbf{r}_{s}\right) \approx \gamma_{b} U_{s}(0),
$$

$$
\begin{aligned}
\rho_{s}\left(\mathbf{r}_{s}\right) & =\left|U_{s}(0)\right|{ }^{2}\left[\gamma_{a}{ }^{2}+\gamma_{b}{ }^{2}-\left(\gamma_{b}+\lambda \gamma_{a}\right)^{2}\right] \\
& \approx\left|U_{s}(0)\right|^{2}\left(\gamma_{a}-\lambda \gamma_{b}\right)^{2} .
\end{aligned}
$$

Substituting the relations Eqs. (5), (6), (9), and (10), we have

$$
\rho_{8}\left(\mathbf{r}_{\varepsilon}\right)=\left|U_{s}(0)\right|^{2}\left[S_{v \varepsilon}\left(\frac{H_{v v}-K_{v s}}{H_{v v}-E_{c}}\right)-\lambda S_{0 s}\left(\frac{H_{00}-K_{0 s}}{H_{00}-E_{c}}\right)\right]^{2} .
$$

This result shows that there are two electron-transfer processes causing the shfs. The first, represented by the term $\gamma_{a}$ in Eq. (16), arises from the transfer of the impurity $d$ electron to the ligand orbital or, in other words, from the formation of antibonding MO $\psi_{a}$. The second process, involving $\lambda \gamma_{b}$ in Eq. (16), comes from the transfer of ligand electron into impurity ion orbital or, in other words, from the mixture of higher energy configuration $\Psi_{2}$.

It can be shown by perturbation theory that $\lambda$ is proportional to and of the order of magnitude as the

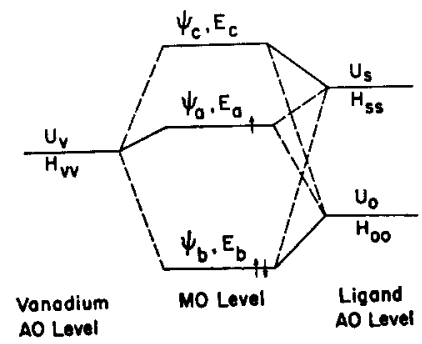

Fig. 1. Schematic energy levels of vanadium, oxygen, and tin atomic orbitals and the molecular orbitals constructed by the linear combinations of the atomic orbitals.

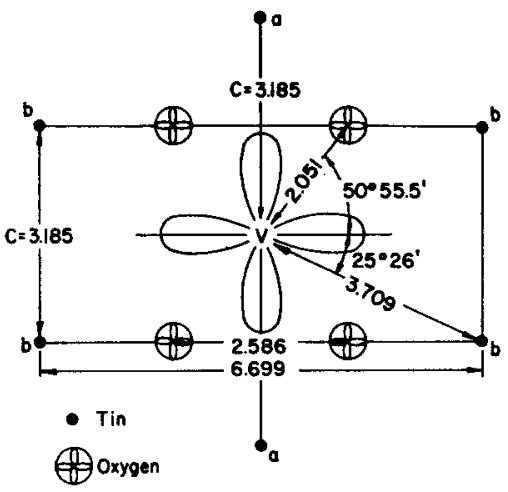

FIG. 2. Diagonal plane of $\mathrm{SnO}_{2}$ unit cell, showing the positions of $a$ and $b$ tins with respect to the substitutional vanadium ion and its $3 d\left(x^{2}-y^{2}\right)$ orbital.

overlap integral $S_{v 0}$, making the second electron transfer process one order of magnitude smaller than the first. Also, Eq. (17) shows that the first process is proportional to the square of the overlap integral $S_{v 8}$.

\section{DISCUSSION}

To compare the above result with experiment the vanadium and tin orbital overlap integrals $S_{v s}$ were calculated. Figure 2 shows the atoms in the diagonal plane of tin oxide unit cell. The two tins on the $c$ axis closest to the vanadium ion are called " $a$ " tins, and the four tins at the corners of the unit cell are the " $b$ " tins. The large and small shfs (see Table I) observed by From $e t a l .{ }^{5}$ are attributed to the interaction between unpaired electron and $a$ - and $b$-tins, respectively. Further, it has been shown that the ground-state wavefunction consists mainly of $3 d_{\left(x^{2}-y^{2}\right)}$.

The overlap integrals of the vanadium $3 d_{\left(x^{2}-y^{2}\right)}$ and the $5 s$ orbitals of $a$ and $b$ tins are given in Table II. For the radial functions of the vanadium $3 d$ orbitals, the Slater function

$$
\psi_{\text {slater }}(3 d)=\phi_{3}(1.43)
$$

and the Hartree-Fock function ${ }^{8}$

$$
\begin{aligned}
\psi_{\mathrm{HF}}(3 d)=0.5243 \phi_{3}(1.83)+0.4989 \phi_{3}(3.61) \\
+0.1131 \phi_{3}(6.80)+0.0055 \phi_{3}(12.43)
\end{aligned}
$$

were used for the neutral vanadium, and

$$
\psi_{\text {slater }}(3 d)=\phi_{3}(1.67)
$$

for $\mathrm{V}^{4+}$. For the tin $5 s$ orbitals only the Slater function

$$
\psi_{\text {slater }}(5 s)=\phi_{4}(1.412)
$$

was used since the Hartree-Fock function is not available. In the above expressions

with

$$
\phi_{n}(\mu)=N_{n \mu} r^{n-1} e^{-\mu r},
$$

$$
N_{n \mu} \equiv\left[(2 \mu)^{2 n+1} /(2 n) !\right]^{1} .
$$

${ }^{8}$ R. E. Watson, Phys. Rev. 119, 1934 (1960). 
TABLE I. Results of EPR experiment on $\mathrm{SnO}_{2}: \mathrm{V}^{4+}$.

\begin{tabular}{lccc}
\hline & $x$ & $y$ & $z$ \\
\hline$g$ & 1.939 & 1.903 & 1.943 \\
hfs $A$ (gauss) & 23.3 & 47.03 & 154.4 \\
$\operatorname{shfs} a$ (gauss) & $\sim 166$. & 172.6 & 165.2 \\
shfs $b$ (gauss) & $\sim 28$ & 28 & 28 \\
\hline \hline
\end{tabular}

It is to be noted that the calculated values of the ratio of the square of the overlap integrals are all close to the experimental value of 6 , which is the ratio of the $a$-tin to the $b$-tin shfs.

The overlap integrals of vanadium and nearest ligand oxygen orbitals are also given in Table II. These results provide a justification for the earlier assumption that all overlap integrals in this complex are of the same order of magnitude.

The isotropic $a$-tin shfs observed for $\mathrm{Cr}$ and $\mathrm{Mn} 3 d^{3}$ configurations, 38 and $31.3 \mathrm{G}$, respectively, ${ }^{9}$ compared to the $168 \mathrm{G}$ for the $\mathrm{V} 3 d^{1}$ configuration can also be accounted for within the framework of the theory presented here. The isotropic shfs constant can be written in the form

$$
A_{s}{ }^{n}=(1 / 2 S) \frac{8}{3} \pi g_{e} \beta_{e} g_{n} \beta_{n} \rho_{s}\left(\mathbf{r}_{n}\right),
$$

where $S$ is the total electron spin and $\rho_{s}\left(\mathbf{r}_{n}\right)$ is the unpaired spin density at the ligand nucleus $\mathbf{r}_{n}$, given in Eq. (17).

Assuming that the orbital energies $H, E, K$ do not change much in going from $\mathrm{V}$ to $\mathrm{Mn}$, we expect that the shfs constants for $\mathrm{SnO}_{2}: \mathrm{V}^{4+}, \mathrm{SnO}_{2}: \mathrm{Cr}^{3+}$, and $\mathrm{SnO}_{2}: \mathrm{Mn}^{4+}$ to be proportional to the square of the overlap integrals between the corresponding metal orbitals and the tin $5 s$ orbital and inversely proportional to the total electronic spin $S$. Calculated results show good agreement with experimental data, (Table III).

Overlap integral between $\mathrm{V} 3 d_{x^{2}-y^{2}}$ and the $5 s$ of $a^{\prime}$ tin (the tin on the $c$ axis with distance $2 c$ away from $V$ ) is found to be about $1 \%$ of that between $\mathrm{V}$ and the

TABLE II. Overlap integrals in $\mathrm{SnO}_{2}: \mathrm{V}^{4+}$.

\begin{tabular}{lccc}
\hline \hline \multicolumn{1}{c}{$\begin{array}{c}\text { Vanadium } \\
\text { orbital }\end{array}$} & $\begin{array}{c}\text { Hartree-Fock } \\
\text { V (neutral) }\end{array}$ & $\mathrm{V}$ (neutral) & $\mathrm{V}^{4+}$ \\
\hline$S_{v 0}\left(x^{2}-y^{2} \mid s\right)$ & -0.01932 & & \\
$S_{v 0}\left(x^{2}-y^{2} \mid x\right)$ & 0.06652 & & \\
$S_{v 0}\left(x^{2}-y^{2} \mid y\right)$ & -0.02805 & & \\
$S_{v s}\left(x^{2}-y^{2} \mid a\right)$ & -0.04212 & -0.1313 & -0.0910 \\
$S_{v s}\left(x^{2}-y^{2} \mid b\right)$ & 0.01640 & 0.0583 & 0.0379 \\
{$\left[S_{v s}(a) / S_{v s}(b)\right]^{2}$} & 6.60 & 5.08 & 5.76 \\
\hline \hline
\end{tabular}

${ }^{9}$ W. H. From, C. Kikuchi, and P. Dorain, Phys. Rev. 135 , A 710 (1964). $a$ tin. The shfs due to $a^{\prime}$ tin will be smaller than that due to $a$ tin by a factor of $10^{-4}$ and seems to be impossible to observe.

In their treatment of shfs in $\mathrm{ZnF}_{2}: \mathrm{Mn}^{4+}$, Marshall and Stuart ${ }^{10}$ obtained a reasonable agreement with experimental value of isotropic shfs by using the HeitlerLondon model (i.e., the mixing coefficient is given by the overlap integral between the metal and ligand orbitals) and considering only the admixture of the fluorine $2 s$ function. But the agreement disappears with the inclusion of the fluorine $1 s$ function. They explained this disagreement as due to the difficulty in computing exactly the overlap integral between Mn $3 d$ orbital and F $1 s$ orbital because of the distortion of $\mathrm{Mn} 3 d$ orbital in the neighborhood of $\mathrm{F}$ nucelus where $\mathrm{F} 1 s$ has quite a large amplitude. They asserted that the contribution from $F 1 s$ orbital to isotropic shfs, if properly calculated, should be small.

In the present work, the contributions from the ligand inner core orbitals can be taken care of by

\begin{tabular}{lccc}
\multicolumn{4}{c}{ TABLE III. Shfs of $\mathrm{SnO}_{2}: \mathrm{V}^{4+}\left(\mathrm{Cr}^{3+}, \mathrm{Mn}^{4+}\right)}$. \\
\hline & $\mathrm{SnO}_{2}: \mathrm{V}^{4+}$ & $\mathrm{SnO}_{2}: \mathrm{Cr}^{3+}$ & $\mathrm{SnO}_{2}: \mathrm{Mn}^{4+}$ \\
\hline shfs (gauss) & 168 & 38 & 31.3 \\
Spin $(S)$ & $\frac{1}{2}$ & $\frac{3}{2}$ & $\frac{3}{2}$ \\
(shfs) $\cdot(2 S)$ & 168 & 114 & 93.9 \\
Ratio & 1 & 0.678 & 0.5589 \\
Overlap & -0.04212 & -0.03493 & -0.02904 \\
(Overlap) $^{2}$ & 0.001784 & 0.00122 & 0.000844 \\
Ratio & 1 & 0.6839 & 0.4728 \\
\hline \hline
\end{tabular}

replacing the outermost $S$ orbital density at the nucleus $\left|U_{s}(0)\right|^{2}$ by the amount of spin polarization with one electron in the outermost $s$ orbital.

Unrestricted Hartree-Fock calculations of this quantity in lithium by Sachs ${ }^{11}$ and Goodings ${ }^{12}$ give about $30 \%$ more than the density of $2 s$ electron at the nucleus obtained from restricted Hartree-Fock calculations. If the trend is the same in fluorine, this replacement will give a better agreement with experiment for the fluorine shfs than that Marshall and Stuart obtained by considering only $2 s$ electron, and at the same time the contribution from $1 s$ electron is taken care of without the necessity of knowing the distortion of $d$ orbital near the $\mathrm{F}$ nucleus.

\section{ACKNOWLEDGMENTS}

We wish to express our thanks to Dr. Geza L. Gyorey for his assistance in the use of digital computer. Discussions with colleague S. Karavelas are also appreciated.

\footnotetext{
${ }^{10}$ W. Marshall and R. Stuart, Phys. Rev. 123, 2048 (1961).

${ }^{11}$ L. M. Sachs, Phys. Rev. 117, 1504 (1960).

${ }^{12}$ D. A. Goodings, Phys. Rev. 123, 1706 (1961).
} 